\title{
Measurement of the $\mathrm{X}(3872)$ production cross section via decays to $J / \psi \pi^{+} \pi^{-}$
}

\author{
Martino Dall'Osso* on behalf of the CMS Collaboration \\ INFN \& University of Bologna and Padova \\ E-mail: dallosso@pd.infn.it
}

The production of the $\mathrm{X}(3872)$ is studied in $p p$ collisions at $\sqrt{s}=7 \mathrm{TeV}$ with the CMS detector at LHC, using decays to $J / \psi \pi^{+} \pi^{-}$where the $J / \psi$ decays to two muons. The cross-section ratio with respect to the $\psi(2 S)$ and the fraction of $\mathrm{X}(3872)$ coming from B-hadron decays are measured as a function of transverse momentum $\left(p_{T}\right)$, covering unprecedentedly high values of $p_{T}$. For the first time, the prompt $\mathrm{X}(3872)$ cross section times branching fraction is extracted differentially in $p_{T}$ and compared with NRQCD predictions. The dipion invariant-mass spectrum of the $J / \psi \pi^{+} \pi^{-}$system in the $\mathrm{X}(3872)$ decay is also investigated.

14th International Conference on B-Physics at Hadron Machines April 8-12, 2013

Bologna, Italy

${ }^{*}$ Speaker. 


\section{Introduction}

The observation of many new states, with masses above the open-charm threshold, that do not fit into the conventional quark model has renewed the interest in exotic quarkonium spectroscopy. The X(3872) was discovered by the Belle experiment in 2003 [1] and, despite a series of detailed studies performed at B-factories and Tevatron, its nature still remains unknown. Some interpretations describe the $\mathrm{X}(3872)$ as a molecular state (loosely bound state of $D^{0 *} \bar{D}^{0}$ ) or as a tetraquark state (bound state of diquark-antidiquark) [2]. At Large Hadron Collider (LHC) there is the opportunity to study this state with high statistics and the measurement of the prompt $\mathrm{X}(3872)$ production rate as a function of transverse momentum can provide a test of the NRQCD factorization approach to $\mathrm{X}(3872)$ production. In this note, the measurement of production of the $\mathrm{X}(3872)$ in $p p$ collisions at a centre-of-mass energy $(\sqrt{s})$ of $7 \mathrm{TeV}$ is presented in Section 2 while a study of the invariantmass distribution of the dipion system in $\mathrm{X}(3872)$ decay is shown in Section 3.

\section{Measurement of the $X(3872)$ production cross section}

The analysis is performed on the data recorded by the CMS experiment in 2011, corresponding to an integrated luminosity of $4.8 \mathrm{fb}^{-1}$. The $\mathrm{X}(3872)$ is observed using the decays into $J / \psi \pi^{+} \pi^{-}$, with the subsequent decay of the $J / \psi$ into a pair of muons. This decay channel has a clean experimental signature in CMS thanks to the high granularity of the tracker and the high efficiency of $J / \psi$ identification. A detailed description of the detector can be found elsewhere [3]. The data are collected with requirements on the dimuon system imposed at the trigger level such as rapidity within 1.25 and a transverse momentum threshold of $9.9 \mathrm{GeV}$. The analysis is thus performed in the kinematic range of $p_{T}$ of the $J / \psi \pi^{+} \pi^{-}$system between 10 and $50 \mathrm{GeV}$ and the rapidity within $|y|<1.2$, collecting about $12000 \mathrm{X}(3872)$ candidates. The event selection and the event simulations, used to determine acceptances and efficiencies, are described in detail in Ref.[4]. The $\mathrm{X}(3872)$ is assumed to be an unpolarized state and its $J^{P C}$ is fixed at $1^{++}$value, as favoured by existing data [2] and confirmed by latest LHCb study [5].

\subsection{Measurement of the cross section ratio}

The ratio of the $\mathrm{X}(3872)$ and $\psi(2 S)$ cross sections times their branching fraction to $J / \psi \pi^{+} \pi^{-}$ is obtained from the measured numbers of signal events, $N_{X(3872)}$ and $N_{\psi(2 S)}$, correcting for the efficiency $(\varepsilon)$ and acceptance $(A)$ estimated from simulations, according to

$$
R=\frac{\sigma(p p \rightarrow X(3872)+\text { anything }) \cdot B\left(X(3872) \rightarrow J / \psi \pi^{+} \pi^{-}\right)}{\sigma(p p \rightarrow \psi(2 S)+\text { anything }) \cdot B\left(\psi(2 S) \rightarrow J / \psi \pi^{+} \pi^{-}\right)}=\frac{N_{X(3872)} \cdot A_{\psi(2 S)} \cdot \varepsilon_{\psi(2 S)}}{N_{\psi(2 S)} \cdot A_{X(3872)} \cdot \varepsilon_{X(3872)}} .
$$

The signal yields, $N_{X(3872)}$ and $N_{\psi(2 S)}$, are determined from unbinned maximum-likelihood fits to the invariant-mass spectra of the $J / \psi \pi^{+} \pi^{-}$system, separately for the $\mathrm{X}(3872)$ and $\psi(2 S)$ in different mass windows. The $\psi(2 S)$ is parametrized using two Gaussian functions with a common mean, while a single Gaussian is used for the $\mathrm{X}(3872)$ signal and the nonresonant background is fitted with a second-order Chebyshev polynomial. The acceptances and efficiencies of the X(3872) and $\psi(2 S)$ final states are determined from the simulation. Studies on Monte Carlo are performed to determine the systematic uncertainties and a data-driven method is used to verify the efficiencies, 
as described in Ref.[4]. The main systematic uncertainty arises from the limited knowledge of the $\mathrm{X}(3872)$ transverse momentum. Both $\mathrm{X}(3872)$ and $\psi(2 S)$ states are considered unpolarized and no systematic uncertainty is assigned to cover other cases. The total uncertainty for each $p_{T}$ interval is typically $10 \%$ statistical and $5-13 \%$ systematic. The results for the ratio of the cross section times branching fraction are shown in Fig.1 (left). No significant dependence on $p_{T}$ is observed.

\subsection{Measurement of the nonprompt fraction}

The fraction of $\mathrm{X}(3872)$ produced from decays of $\mathrm{B}$ hadrons is referred to as nonprompt fraction. The X(3872) candidates from B hadron decays are selected based on the pseudo-proper decay lenght $\left(l_{x y}\right)$, defined in Ref.[4] and related to the distance between the primary vertex and the secondary one, formed by the $J / \psi \pi^{+} \pi^{-}$system. A B-hadron-enriched sample is obtained requiring $l_{x y}$ larger than $100 \mu m$, with an estimated contribution from prompt $\mathrm{X}(3872)$ below $0.1 \%$. The nonprompt fraction is then obtained from the ratio between the signal yields extracted from this B-hadron-enriched sample and the signal yields in the inclusive sample, after correction for the efficiencies of the decay-length-selection criteria determined from simulations. Detailed studies are performed to verify the stability of the results and to determine the systematic uncertainties leading to a total systematic uncertainty of $6-10 \%$, as described in Ref.[4]. The measurement is dominated by its statistical uncertainty $(\sim 20 \%)$. The X(3872) nonprompt fraction reveals no significant dependence on transverse momentum, as shown in Fig.1 (right). These results are obtained assuming null polarization for the $\mathrm{X}(3872)$ and no systematic uncertainty is assigned.
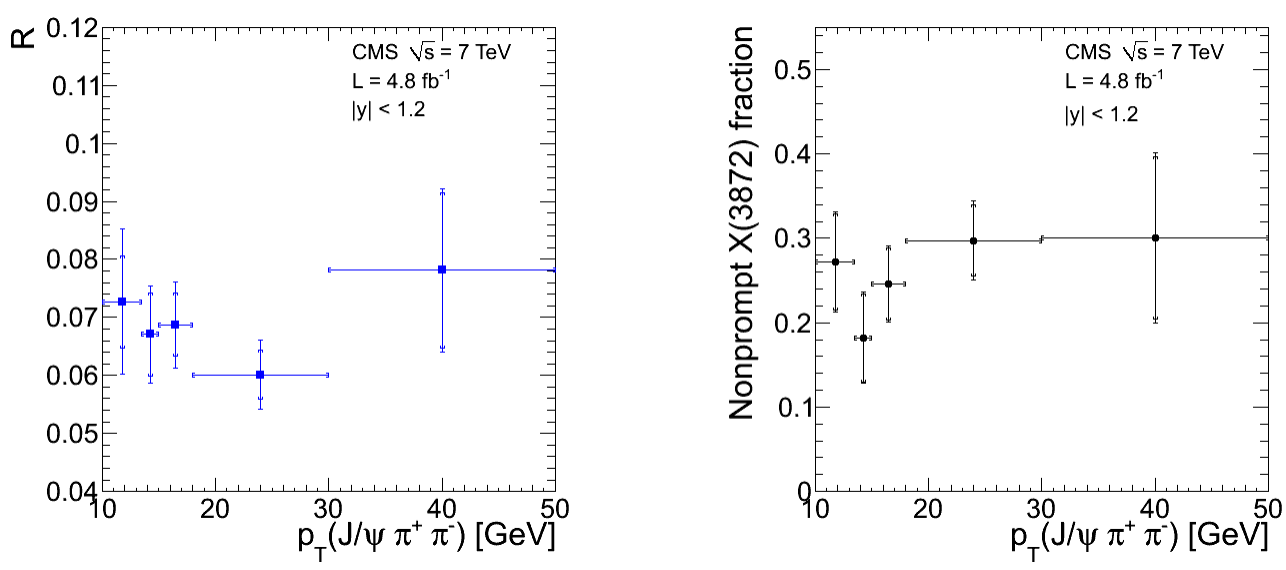

Figure 1: Ratio between $\mathrm{X}(3872)$ and $\psi(2 S)$ cross sections times branching fraction (left) and $\mathrm{X}(3872)$ nonprompt fraction (right), as a function of $p_{T}$. Outer error bars are the total uncertainty while inner error bars indicate only statistical uncertainty. The data points are placed at the centre of each $p_{T}$ bin.

\subsection{The prompt $X(3872)$ production cross section}

The cross section times branching fraction for prompt $\mathrm{X}$ (3872) production is determined from the measurement of the cross section ratio $R$ and the nonprompt fraction, described above, combined with a previous result of the prompt $\psi(2 S)$ cross section obtained in CMS [6]. The latter measurement was performed using the $\psi(2 S) \rightarrow \mu^{+} \mu^{-}$decay mode and provides results as a function of transverse momentum up to $30 \mathrm{GeV}$, in the same rapidity range of this analysis. By means 
of this combination, the differential cross section for prompt $\mathrm{X}(3872)$ production times the branching fraction is obtained as a function of $p_{T}$, in the rapidity region $|y|<1.2$, as shown in Fig.2. The $\mathrm{X}(3872)$ and $\psi(2 S)$ states are assumed to be unpolarized and no cancellation of systematic uncertainties is assumed in the combination. The main sources of systematic uncertainty are related to the measurement of $R$ and of the prompt $\psi(2 S)$ cross section [6]. The differential cross section for prompt $\mathrm{X}(3872)$ production in $p p$ collisions at $\sqrt{s}=7 \mathrm{TeV}$ has also been predicted with a calculation made within the NRQCD factorization formalism [7]. In this calculation the cross section is expressed as the sum of parton cross sections for creating $c \bar{c}$ pairs with vanishing relative momentum multiplied by phenomenological constants and results are normalized using Tevatron measurements with the statistical uncertainty obtained from the experimental input data. The predictions from Ref.[7] were modified by the authors to match the phase-space of the measurement presented in this note. The comparison of this prediction with the data is shown in Fig.2 and demonstrates that, while the shape is reasonably well described, the predicted cross section is much larger than the measured one. The integrated prompt $X(3872)$ cross section times branching fraction for the kinematic region $10<p_{T}<30 \mathrm{GeV}$ and $|y|<1.2$ is also determined to be $\sigma^{\text {prompt }}(p p \rightarrow X(3872)+$ anything $) \cdot B\left(X(3872) \rightarrow J / \psi \pi^{+} \pi^{-}\right)=1.06 \pm 0.11($ stat. $) \pm 0.15($ syst. $) n b$.

This measured value is significantly below the NRQCD prediction for the prompt $\mathrm{X}(3872)$ cross section times branching fraction in the same kinematic region, which is $4.01 \pm 0.88 \mathrm{nb}$ [7].

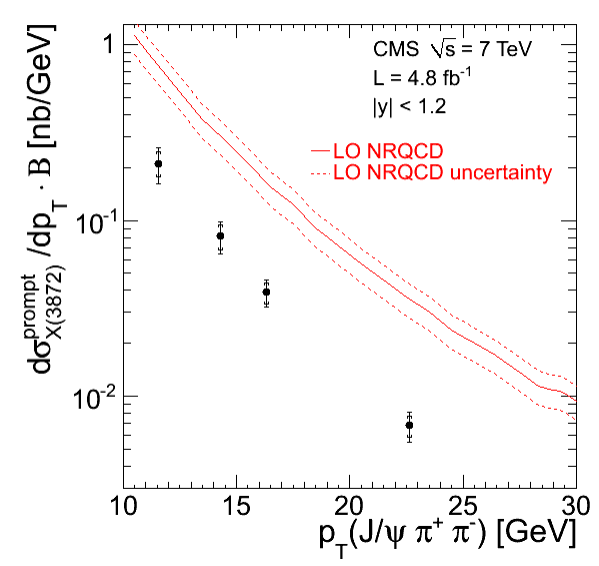

\section{Study of the $\mathrm{X}(\mathbf{3 8 7 2})$ decay properties}

The dipion invariant-mass distribution from $\mathrm{X}(3872)$ decays to $J / \psi \pi^{+} \pi^{-}$is measured in order to investigate the decay properties of the X(3872) and evaluate the presence of an intermediate $\rho^{0}$ state in the decay, as suggested by previous studies [8]. The measurement is made within the kinematic region $10<p_{T}<50 \mathrm{GeV}$ and $|y|<1.25$. To extract the dipion invariant-mass spectrum from $\mathrm{X}(3872)$ decays, the event sample is divided into intervals of $m\left(\pi^{+} \pi^{-}\right)$in the range $0.5-0.78$ $\mathrm{GeV}$. In each interval, a maximum-likelihood fit to the $J / \psi \pi^{+} \pi^{-}$invariant-mass distribution is performed with the signal modelled by a Gaussian. The position and width of the $\mathrm{X}(3872)$ signal are fixed to the values obtained in the fit to the full sample. The dipion invariant-mass distribution is 
extracted from the signal yields obtained from these fits to the data in each interval, after correction for detector acceptance and efficiency estimated from the simulation, as described in Ref.[4]. The main systematic uncertainties are related to the signal extraction $(10-20 \%)$ and to corrections due to acceptance and efficiency $(4-6 \%)$. The resulting dipion invariant-mass spectrum, normalized to the total cross section in the interval $0.5<m\left(\pi^{+} \pi^{-}\right)<0.78 \mathrm{GeV}$, is presented in Fig.3. The data are compared to $\mathrm{X}(3872)$ signal simulations with and without an intermediate $\rho^{0}$ in the $\mathrm{X}(3872)$ decay. The assumption of an intermediate $\rho^{0}$ decay gives better agreement with the data.

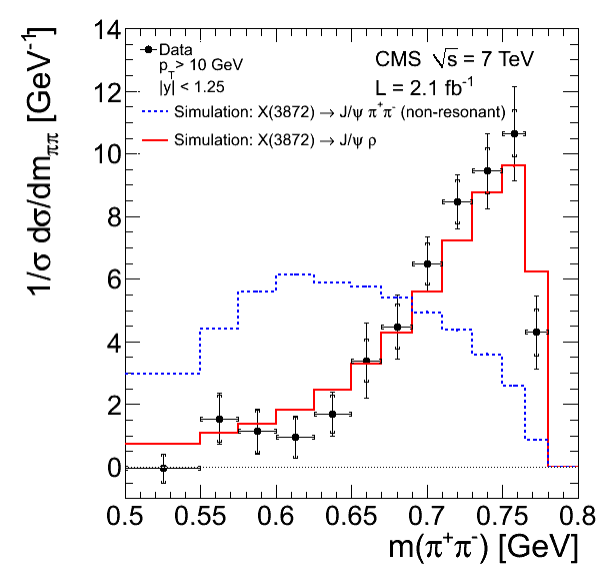

Figure 3: Dipion invariant-mass spectrum for $X(3872) \rightarrow J / \psi \pi^{+} \pi^{-}$decays corrected for acceptance and efficiency. The distribution is normalized to unity by dividing by the total cross section for $0.5<$ $m\left(\pi^{+} \pi^{-}\right)<0.78 \mathrm{GeV}$. The inner error bars indicate the statistical uncertainty and the outer one represent the total uncertainty. The result is compared to spectra from simulation with (solid line) and without (dotted line) an intermediate $\rho^{0}$ decay.

\section{Conclusions}

The $\mathrm{X}(3872)$ production cross section is measured in $p p$ collisions at $\sqrt{s}=7 \mathrm{TeV}$ with data collected by the CMS experiment in 2011. The X(3872) and $\psi(2 S)$ are observed using their decay into $J / \psi \pi^{+} \pi^{-}$. The ratio of their cross sections times branching fractions and the fraction of $\mathrm{X}(3872)$ from B-hadron decays are measured. Both do not show significant dependence on $p_{T}$. Using these measurements, for the first time the prompt $\mathrm{X}(3872)$ production cross section is obtained as a function of $p_{T}$. The available NRQCD predictions significantly overestimate it while the dependence on $p_{T}$ is reasonably described by the theory. The study on the dipion mass spectrum for $X(3872) \rightarrow J / \psi \pi^{+} \pi^{-}$favours the presence of an intermediate $\rho^{0}$ state in the X(3872) decay.

\section{References}

[1] Belle Collaboration 2003, Phys. Rev. Lett. 91262001.

[2] N. Brambilla et al. 2011, Eur. Phys. J. C 711534.

[3] CMS Collaboration 2008, JINST 3 S08004.

[4] CMS Collaboration 2013, JHEP 04 (2013) 154.

[5] LHCb Collaboration 2013, Preprint arXiv:1302.6269.

[6] CMS Collaboration 2012, JHEP 02 (2012) 011.

[7] P. Artoisenet and E. Braaten 2010, Phys. Rev. D 81114018.

[8] CDF Collaboration 2006, Phys. Rev. Lett. 96102002. 